This is the post print version of the article, which has been published in Science as culture. 2018, 27 (4), 464-487. http://doi.org/10.1080/09505431.2018.1508429

D) This document has been downloaded from TamPub.tafif

a The Institutional Repository of University of Tampere

\title{
Tuning Clinical Recruitment around Cultural Taboos in a Human Microbiome Study
}

\author{
Elina I. Mäkinen* \\ New Social Research and Faculty of Management \\ University of Tampere
}

\begin{abstract}
(207 words)
Human microbial communities are bodies of microorganisms that reside on different body parts. Importantly, they have been found to affect human health. Scientific research on human microbial communities has created new challenges for human subject recruitment. First, individuals are asked to collect samples of bodily substances that can be seen as repulsive (e.g., feces and urine). Second, because scientists want to understand how human microbial communities evolve over time, individuals are asked to commit to a regular sample collection for extended periods of time. A longitudinal qualitative study of the work of scientists, physicians, research staff, and study coordinators involved in a human microbiome research project has found that these actors can bypass some aspects of recruitment and retention challenges through tuning work. Tuning work is a collaborative process where professionals agree to adjust their practices towards shared goals. The research actors reconfigured their work practices, personal routines, and the study protocol in an effort to ease the obstacle created by cultural taboos against handling bodily substances. The burden of long-term participation provided fewer opportunities for tuning work for the study coordinators and the physicians. The scientists, in turn, refused to modify the extensiveness of the protocol. Therefore, long-term commitment became a permanent recruitment and retention obstacle.
\end{abstract}

\section{Keywords}

Human microbiome, human subject recruitment, recruitment practices, cultural taboo, fecal habitus, tuning

*Correspondence Address: Elina I. Mäkinen, Faculty of Management, 33014 University of Tampere, Finland. Email: elina.i.makinen@uta.fi 


\section{Introduction}

$23 \& \mathrm{Me}$ and Ancestry.com are companies that provide individuals an opportunity to know more about their ancestry and health. These companies sell kits that can be used to collect saliva samples. The saliva samples are sent back to the companies, where they go through a genealogical DNA testing. The results can reveal varied details about the person's family history or health related factors.

A more recent addition to these types of companies is uBiome, which sells kits for selfsampling microbial communities living in gut or vagina. Human microbial communities are bodies of microorganisms that reside on different body parts and that have been found to affect human health. uBiome has developed a sequencing-based clinical microbiome test that allows for an analysis of microbial flora and its impact on different health factors. The test for gut samples - fecal matterprovides information of microbes and explains their impact on health, while the test for vaginal samples reveals results relating to HPV, STIs, and vaginal flora.

One of the editors at Popsugar.com sent her fecal sample to uBiome for microbial analysis and wrote about her experience in an editorial (Gabillet, 2017). Referring to the cultural taboo around fecal matter, she said, 'Assuming you're comfortable collecting and sending a small sample of your stool (i.e. poop), uBiome will analyze your microbes and present you actionable results.' When explaining the actual sample collection procedure, and comparing it to $23 \& \mathrm{Me}$ and Ancestry.com, she said: 'There's a catch, though: instead of swabbing the inside of your month with a Q-tip or spitting into a little tube to collect your DNA, you have to dab a Q-tip on a piece of soiled toilet paper. The directions promise they only need a tiny amount, so it's actually not that gross.'

The good news for the customers is that uBiome can work with only a onetime sample. In universities, however, human microbiome researchers collect several samples of bodily substances from the same individual in order to understand how changes in human microbial communities influence health. Therefore, the study of human microbial communities has created new challenges 
for human subject recruitment. Research subjects are asked to collect monthly, weekly, or sometimes even daily samples of bodily substances that many perceive as embarrassing — such as feces and urine - and to make the sample collection procedure a routine part of their lives. This leads to the question, how do research actors moderate feelings of embarrassment and intrusion resulting from cultural taboos and the longitudinal nature of human microbiome studies?

This longitudinal qualitative study examines how a heterogeneous group of research actorsscientists, physicians, research staff, and study coordinators - sought to overcome obstacles to human subject recruitment in the context of human microbiome research. I draw on Pickering's (1995) concept of tuning to explain how the research actors adjusted their individual work practices in an effort to achieve the shared recruitment goals.

Pickering's $(1993 ; 1995)$ conceptualization of 'dance of agency' was intended to describe the constant accommodation and resistance taking place between scientists and their machines as they create scientific knowledge. In human microbiome research, before scientists can use instruments to analyze human microbial specimens, they must engage in human-to-human interaction to gather the samples they need. Just as technological instruments may interrupt scientific processes, study participants can hamper the conduct of science by not performing the way research actors want. This is particularly likely when research subjects are asked to handle polluted matter-such as fecestypically perceived as taboo (Douglas, 1966). Yet only a very few sociological studies have examined how scientists gather data on substances that research participants experience in this way.

The paper unfolds as follows. I first discuss how Pickering's (1995) conceptualization of 'tuning' advances our understanding of collaborative work in the context of human subject recruitment. Second, I demonstrate how the concepts of 'fecal habitus' and 'cultural taboo' can be used to explain what makes human subject recruitment for the purposes of human microbiome research particularly challenging (Douglas, 1966; Inglis, 2000; Weinberg and Williams, 2005). Next, 
I describe the research setting, data collection, and analytical approach. The empirical analysis reveals, first, how the research actors characterized the recruitment obstacles relating to human microbiome research and, second, how they addressed them through tuning work. I conclude by summarizing the main findings and discussing how Pickering's (1995) concepts about the relationship between human and non-human actors are applicable in the study of collaborative work among research actors recruiting subjects to a human microbiome study.

\section{Tuning Human Subject Recruitment}

Human subject recruitment in biomedical research brings together research actors-for example, scientists, physicians, nurses, and research staff—who need to figure out how to align their work practices with shared recruitment goals (Mueller, 1997). Pickering's (1995) The Mangle of Practice: Time, Agency, and Science offers analytical tools for understanding the adjustments that need to be made among a heterogeneous group of research actors as they seek to recruit research subjects.

Pickering (1995) argued that material objects, such as scientific instruments and machines, can resist and interrupt scientific experiments. When scientists encounter problems using technological equipment, they adjust to them by revising their goals and practices. As a result, the technological and the social interactively stabilize one another through a process of trial and error in a dance of agency. To explain this process, Pickering used the concept of tuning, which can be defined as the reciprocal adjustment of human and material agency. Tuning describes how the technological and the social 'become part of a unitary, but heterogeneous, assemblage' (Pickering, 2005, p. 365).

Pickering's dance of agency has been applied in a range of different contexts to further understanding of the relationship between non-human and human actors. Griswold et al. (2013) studied two museum exhibitions to show how interactions between non-human agents-such as 
material objects and words_-and exhibition visitors generated the art encounter. In the context of the food industry, Martini et al. (2013) examined a social media platform that was meant to engage external actors in the firm's innovation processes. The researchers considered how the social media platform's material agency configured and reconfigured different modes of engagement among the firm's employees and customers. In a different field, Brenninkmeijer (2013, p. 146) observed work practices in neurofeedback clinics and illustrated how human and non-human actors 'struggle, collaborate, and swap roles in a process that creates a new self for the neurofeedback client'.

A study by Barrett et al. (2012) also drew on the mangle of practice, but instead of focusing on the interactions between human and non-human actors, it used the concept of tuning to explain how the installation of a robot shaped working relations between three occupational groups in a hospital pharmacy. The study highlighted how a technological change transformed boundary relations, professional jurisdictions, skills, and status among pharmacists, technicians, and assistants. These three interdependent occupational groups then had to reconfigure their ways of working together.

In a similar manner, a human subject recruitment effort brings together different professionals who need to tune their individual work practices towards shared goals. As Pickersgill (2012, p. 584) has noted, 'participant recruitment is seldom an easy task and it can have significant implications for the nature of the study'. How scientists are able to attract subjects to their studies can impact the reliability of scientific discoveries. In the process of generating scientific findings, actors are assigned different social roles that determine how they can participate in knowledge creation (Callon, 1986; Latour, 1987; Lappé, 2014). Even at the stage of drafting study protocols, research actors compose documents that they then use to guide their own and others' actions. Scientists, for example, may imagine human subjects as abiding participants committed to given study protocols. 
Human subject recruitment study protocols are not 'immutable mobiles,' however (Latour, 1990). Much like laboratory manuals, work practices and written protocols intertwine and supplement each other' (Lynch, 2002, p. 210). Moreover, as human subjects are independent actors, they have interests that do not necessarily align with those of the scientist (Epstein, 1997; Oudshoorn, 2003). Compared to other research material, human beings can 'talk back' and 'vote with their feet' (Epstein, 2008, p. 806). Scientists may even fear failing in their human subject recruitment efforts. In Pickersgill's (2012, p. 585) study of the discourses surrounding ethical research, a neuroscientist proclaimed, '[I]f you need to get subjects from the general public, you know, you need these tricks'.

The development of recruitment tricks may be particularly imperative when research subjects are asked to deal with embarrassing and private bodily substances. A number of natural functions such as defecating, urinating, and spitting have historically been public acts but, over time, they have been eliminated from social life (Elias, 2000). Bodily secretions like sweat, tears, urine, and feces are now dealt with in privacy. They take place behind the scenes rather than in public (Synnott, 1993; Cahill, 2006). Moreover, negative perceptions of bodily excretions like feces and vomit are not only present in Western societies; rather, they are considered to be repulsive across all cultures (Rozin and Fallon, 1987; Curtis and Biran, 2001).

Fecal material, specifically, is considered to be a 'universal disgust substance' (Rozin et al., 1993, p. 579). In order to manage this disgust substance, individuals embody a fecal habitus that helps them with organizing their bodies and following the everyday social order regulating fecal outputs (Inglis, 2000; Weinberg and Williams, 2005). Inglis (2000) has identified two components in this habitus. First, both morally and hygienically, excrement must be classified as dirt. Second, individuals must take into account excretory practices that relate to correct times and locations for defecation, sensory considerations like sound and smell, and how excrement is talked about. 
The highly emotional, negative meanings assigned to certain bodily substances speak to the taboo nature of the matter that human microbiome researchers wish to study. Taboos are rules about our behavior that restrict how we interact with things, objects, and people (Douglas, 1966). There are symbolic boundaries that define some material things and actions as admissible and some as taboo (Epstein, 1992). Issues that fall outside of what is perceived as acceptable become morally reprehensible. They may be regarded as pollution, dirt, or 'matter out of place' (Douglas, 1966, p. 36). Douglas (1966) emphasized that dirt is not an isolated event, but part of a larger system of how we order and classify matters. The system of rituals around purity and impurity can even create a unity of experience.

If individuals are forced to interact with controversial matter, they become anxious and fearful about becoming polluted. In these instances, the order of things is challenged, and individuals need to figure out how to reestablish it through particular activities and rituals (Goffman, 1967). At the same time, whether handling particular matter is perceived as a taboo or not and whether it causes negative emotions will depend on who is making the judgment. As Douglas (1966, p. 2) pointed out, 'there is no such thing as absolute dirt: it exists in the eye of the beholder'. Study participants and medical researchers, for example, are likely to perceive the taboo nature of fecal matter differently.

Very few sociological studies have explored how taboos influence human subject recruitment and medical research. A rare example is Thompson's (2013) study of inflammatory bowel disease support groups. He discovered that stool sample tests created anxiety in people suffering from the disease: they believed that dealing with stool samples was socially damaging; they feared that they might physically pollute their environment; and they were afraid that others might find out what they were doing. Relatedly, some medical researchers have assessed low uptake for bowel cancer screening programs. These studies found that screening-program participants felt there was a cultural taboo against sampling and storing feces (Palmer et al., 2014; Palmer et al., 2015). 
This suggests that if research actors wish to collect data on bodily substances that subjects experience as potentially taboo, they need to moderate feelings of embarrassment and intrusion. This study examines how research actors engaged in tuning work and adjusted their work practices in an effort to ease recruitment and retention obstacles common in human microbiome research. Before turning to the empirical findings, I describe the data and how they were analyzed.

\section{Research Design}

The data for this study were collected as part of a longitudinal ethnographic research project involving a new medical research center located in a school of medicine in the United States. The center focused on studying pregnancy-related complications, such as premature birth. The present longitudinal qualitative case study examines one of the center's scientific teams. The team wanted to understand how patterns of change in pregnant women's microbial communities were associated with early delivery and other complications. In collaboration with physicians, research staff, and study coordinators, the scientists planned and implemented a sample collection effort. Specifically, the research actors wanted to recruit 3,000 women planning to get pregnant or whose pregnancy was in its early stages to weekly self-sample oral (saliva sample and tooth or gum swab), gastrointestinal (fecal sample or rectal swab), vaginal (swab), and skin surface (swab) specimens over the course of pregnancy and for several months after delivery.

I began collecting data when the human microbiome team began its work and continued to track the team's evolution for almost three years. The analysis draws on observations and audio recordings from two different types of meetings where the different research actors interacted. All the audio recordings from meetings were transcribed by a transcription service, and I then identified speakers in each of the transcripts. 
First, I observed and recorded four team meetings where the microbiologists and the immunologists leading the project presented their work to other scientists affiliated with the medical research center. Second, I observed and recorded 31 meetings where the team planned a human subject recruitment effort in collaboration with physicians, research staff, and study coordinators. These meetings became my primary source of data. I also draw on secondary sources of data, such as the initial research proposal to form the medical research center and its scientific teams and meeting notes taken by the center administrators.

With regards to the human microbiome project, the research proposal explained what the scientists sought to accomplish, how many women they hoped to recruit, and the significance of their work. The meeting notes prepared by the administrators, in turn, helped me to keep track of the different steps in the planning and the implementation of the study protocol and how the recruitment of study participants progressed over time. These secondary sources of data helped me to contextualize the meeting discussions and how they evolved over time. Table 1 summarizes all the data I draw on in the following empirical analysis.

Table 1: Data Sources

\begin{tabular}{|l|l|}
\hline Data Source & $\begin{array}{l}31 \text { in total } \\
\text { Length of meetings: 60-90 min } \\
\text { Length of transcripts: } 38-50 \text { pages }\end{array}$ \\
\hline $\begin{array}{l}\text { Human subject recruitment } \\
\text { planning meetings }\end{array}$ & $\begin{array}{l}\text { 4 in total } \\
\text { Length of meetings: } 60-70 \text { min } \\
\text { Length of transcript: } 35-45 \text { pages }\end{array}$ \\
\hline Microbiome team meetings & $\begin{array}{l}\text { Research proposal (85 pages) } \\
\text { Administrators' meeting notes } \\
\text { (each about 3 pages) }\end{array}$ \\
\hline Secondary sources & \\
\hline
\end{tabular}

The participants in the human subject recruitment planning meetings were microbiologists and immunologists, physicians who both conducted academic medical research and worked in the 
clinic, research staff, and study coordinators hired for the human microbiome sample collection effort. The scientists were mostly men, the physicians included an equal number of men and women, and the research staff and the study coordinators were all women. The study coordinators were much younger than the scientists and the physicians; they were either former nurses or recent college graduates hoping to apply for medical schools. The number of meeting attendees varied between 6 and 14 and the meetings lasted between 60 and 90 minutes. The transcripts for the audio recorded meetings were between 38 and 50 pages long.

I utilized the framework of discourse analysis when analyzing the meeting transcripts. The transcripts were analyzed to identify patterns of discourse that shaped the meeting participants' shared efforts (Goodwin, 2000; Gee, 2005; Sidnell, 2007). I developed analytical codes for understanding the different types of professional roles and perspectives displayed by the meeting participants and how those shaped conversations in meetings. The most salient standpoints were: microbiome standpoint, physician standpoint, study coordinator standpoint, and patient perspective. Although the study participants were not present in the meetings, I coded for instances where the other research actors - typically the research staff and the study coordinators-recounted what they had learned from their interactions with the women (either at the clinic or when picking up samples from their homes). As I do not have direct evidence of the study participants' experiences, I focus on analyzing discussions among the research actors in which they make sense of recruitment obstacles and ways of addressing them.

I also developed codes for the different tasks that the research actors had to complete. These included, for example, the development of the IRB protocol, the creation of a patient information questionnaire, the logistics concerning self-sampling, and the collection, processing, and storage of microbiome specimens. I examined how the research actors' different perspectives were expressed in 
relation to the tasks that needed to be completed. The coding of all the transcripts was done using the Atlas.ti qualitative data analysis software.

\section{Recruitment and Retention Obstacles in Human Microbiome Research}

Scientists who study human bacterial communities know that finding subjects who are willing to donate their bodily substances over a long period of time is challenging. The human microbiome researchers often mentioned a colleague who had grown tired of the difficulties associated with human subject recruitment. He had begun to sample his wife's, his own, and his dog's stool daily, because this practice provided him with a steady supply of data. The human microbiome researchers wanted to demonstrate that, with regard to data collection, it was possible to do more.

Their study protocol had two characteristics that made it challenging for recruitment. First, the protocol asked subjects to self-sample varied bodily substances, such as feces and urine, and store the samples in their freezers until they were picked up by study coordinators. Second, the protocol asked women to commit to the weekly self-sampling procedure for the duration of their pregnancy and for several months after delivery. The length of the study, combined with asking subjects to handle material perceived as taboo, were recruitment obstacles that needed to be moderated.

\section{The Problem of Cultural Taboos}

The scientists were interested in aspects of the human body that can be perceived as culturally taboo (Douglas, 1966). The research protocol included the collection of weekly oral, vaginal, and skin swabbing and weekly fecal, urine, and saliva specimens. Here is how a scientist explained how the weekly self-sampling should operate in practice: 
I think the women should be given a kit that is designated for each week of pregnancy. That kit should have all the materials they need for their one sampling. Those samplings should involve swabbing and putting the swab back into the sterile swab container, putting that back in the bag, and putting it in their freezer. That would be what they should do for all the swabbings. Then they have a container for stool, a container for urine, and a funnel and a small tube for saliva. All that goes in the freezer and that's going to take up a little bit of space. The swabs are easy to store because you can store them upright in a Ziploc or something, but the stool container and the urine container, they might need weekly or biweekly pick up. (ID$5 ; 070611)$

The scientist described the practicalities of the sample collection procedure matter-of-factly. She was not embarrassed by the collection of fecal material. Fecal material was part of her daily work and, in fact, extremely valuable for scientific purposes.

Douglas (1966, p. 2). argued that 'there is no such thing as absolute dirt: it exists in the eye of the beholder'. What was matter of great value for the scientist, was matter out of place for the research subject. Members of the research staff and the study coordinators feared that asking study participants to self-sample and store fecal material was going to turn away potential subjects. As one said, 'I'm just worried about patients saying no, they won't do it-period-if it involves dealing with their poop' (ID-23; 091411). The research staff and the study coordinators understood that they were asking subjects to rethink shared cultural categories, which 'cannot so easily be subject to revision' (Douglas, 1966, p. 40).

Their concerns were confirmed in focus group interviews, where potential subjects objected to self-sampling fecal material and storing the samples next to food items in their freezers. Surveys and focus group interviews conducted to reveal what motivates individuals to enroll are sometimes 
used in the development of human subject recruitment strategies (Epstein, 2008). They are common when scientists suspect that their research design is demanding or when they need to recruit from a specific group of people, such as pregnant women. Based on what a member of the research staff had learned from the focus group discussion, she argued that the collection of fecal material was going to make enrollment extremely difficult:

I'm concerned that the biggest issue for all the patients was the fecal material. That was clearly the biggest issue, not only in handling it, but storing it [in their freezers], so we were figuring out all sorts of different ways to store it. (D-23; 091411)

The focus group participants had explained that they would not be comfortable with collecting their stool. The idea of keeping stool samples in the freezer until the study coordinators picked them up was seen as even more repulsive. One women had recounted that she could not imagine storing a frozen chicken, for instance, next to stool samples.

By requesting that study participants collect their own feces and store the samples in their freezers, the research actors challenged established excretory practices and assumptions about the dangers of polluted matter (Inglis, 2000; Douglas, 1966). Our ideas of polluted matter are based on hygiene, aesthetics, and 'knowledge of pathogenic organisms' (Douglas, 1966, p. 36). How we perceive matter as dirt - and hence consider it taboo - is a relative idea. Douglas (1966, p. 36) provided a number of examples, explaining that 'food is not dirty in itself, but it is dirty to leave cooking utensils in the bedroom' or to leave 'bathroom equipment in the drawing room'. Similarly here, the research actors asked the research subjects to store polluted matter (feces) in a place (freezer) where it did not belong and where it would contaminate pure matter (food). 


\section{The Burden of Long-Term Participation}

In addition to the hurdle of cultural taboos, the study protocol was taxing because it demanded long-term participation. In other words, the subjects were asked to make the self-sampling of matter out of place a routine part of their lives. The length of time was important for the scientists, because they hypothesized that birth outcomes could be explained by changes in human microbial communities across different body sites and over the course of pregnancy. They also suspected that there were meaningful patterns to be identified in how women's microbial communities stabilized after giving birth.

As the proposed study was exploratory — no other human microbiome researcher had sought to collect a dataset as extensive as this one - the scientists were not sure about the ideal study design. They did not know how many months after delivery the women should continue self-sampling or which body sites were the most important for explaining birth outcomes. Because of all these unknowns, the scientists thought that the longer the women were willing to participate the better.

In one of the first meetings, the human microbiome researchers tried to explain to the other research actors why they wanted to gather samples for as long as possible. In essence, they sought to generate a shared understanding of the project that every group — other scientists, physicians, and study coordinators - could agree on. Callon (1986) saw this as part of the process of translation where scientists negotiate a network of relationships and assign different actors particular roles on a shared project.

ID-24: Is there a difference in the way in which a woman recovers, say her vaginal microbiota recovers from pregnancy, depending on whether she just gave birth prematurely or gave birth at term? In this case, all we would want to do is just follow the vaginal 
microbiome for as long as the women are willing to continue in the study. We may find that women are pretty tired of being involved in our study by the time they deliver.

ID-23: Oh my God! These guys are just crazy! All the women in the room go 'what'?

ID-26: And the men in the room go 'what'? [Laughter] (091411)

As the scientists were interested in how human microbial communities evolved during pregnancy and how they stabilized after giving birth, a longitudinal study design was necessary. After the scientist's explanation, the other meeting participants, both women and men, made jokes about the magnitude of the sample collection effort. The scientist acknowledged that there was a chance that research subjects might get 'tired of being involved in our study' and thus not accept their assigned role. After all, when working with human subjects, scientists need to deal with study participants' subjectivity, agency, and varying levels of commitment (Epstein, 2008).

Latour (1987) and Callon (1986) both addressed the notion of alignment in the conduct of scientific work. Latour (1987) discussed the enrollment of different allies and then keeping those allies in line during the construction of scientific facts. In turn, Callon (1986) problematized the alliances between different entities that are established when scientists work together with other actors. Entities with assigned roles and tasks become aligned and integrated into the shared plan in particular ways. The study coordinators challenged the scientists' plan regarding long-term commitment. As one entity, they argued that the commitment requested from the women should not be overly long and saw it as one of the reasons why women might refuse to participate. The scientists argued, however, that once women were enrolled in the study, they would begin to see the selfsampling procedure as one of their regular routines, much like brushing their teeth.

To create this sense of routine, the scientists said that the women should conduct the selfsampling procedure at the same time each week: 'I think we should ask them, if at all feasible, to try 
to assign a certain time of day as their collection time' (ID-24; 091411). Although the scientist said 'we should ask them,' it was the study coordinators' task to ask subjects to self-sample at the same time of day each week. In these negotiations around the issue of long-term commitment, the scientists had more power. They could determine the scope of the sample collection effort and make sure that the study coordinators, who were hired for the project, complied with the plan (Callon, 1986).

After a few months of recruiting patients in the obstetrics and gynecology clinic, the study coordinators reported that they had enrolled 13 subjects, but two or three of them had already dropped out. This meant that the study coordinators may have been right about their critique on the length of study participation time and that they had valid reasons for not accepting the problematization introduced by the scientists (Callon, 1986). Furthermore, after a year into the recruitment process, the study coordinators had enrolled only 36 women. Three and a half years after the recruitment of the first subject, they had enrolled about 250 women. While this number of subjects resulted in an impressive amount of specimens due to the frequency of the self-sampling procedure, it was significantly less than 3,000, which was stated as the initial recruitment goal.

Related to the dropouts, the scientists wanted to know if the women had specified any reasons for quitting the study; they wanted to understand what was hindering retention. One of the study coordinators explained:

The ones that have dropped out, they felt like they were too busy. They dropped after the first month. So I would say that's okay. Honestly, we want people who really want to be in the study, because it's a big commitment. The only reason that we are learning less with these 13 people is that after they give birth there's a period of silence where we don't know what's going on. Then you just have to know how to do the dance with them and like-really, see 
like okay, so how am I going to re-engage you and say 'hey, do you still want to be in the study'? Because after the baby is born, they kind of like disappear. (ID-34; 011812)

The study coordinators were dealing with two groups of dropouts. They were less worried about those who left the study soon after enrollment, as these individuals were not probably ready for the commitment. The second group of potential dropouts were women who had just given birth. As the scientists wanted to understand how women's microbial communities stabilized after delivery, reengaging the women in the study after they had given birth was important.

The moment of delivery was significant for the scientists and the study participants for different reasons. While for the scientists delivery was a fascinating shock to human microbial communities, for the women it marked the end of pregnancy. In that moment, the women began to transition into the state of motherhood (Stern et al., 1998). During transitional states, a person must pass from one position to another. The process of moving from one state to another can be dangerous and influence the person who goes through it in unexpected ways (Douglas, 1966). The study coordinators felt awkward about 'doing the dance' and trying to get in touch with the new mothers, as the previous speaker noted. Since the study coordinators did not know how the delivery had gone and how the women were doing, calling them felt disrespectful.

When planning study protocols and collecting data on human subjects, research actors determine a role for study participants that includes how they should behave (Latour, 1987). Callon (1986) described this stage as problematization; a process through which the system of associations among groups is solidified and roles for each entity are determined. The study coordinators questioned the longitudinal study design and its impact on the study participants. Because the scientists wanted to recruit individuals to a longitudinal study in which they were expected to commit to weekly self-sampling of different bodily substances before and after a significant life change, the 
role they had determined for the research subjects was challenged. After becoming mothers the research subjects' interests diverged from those of the scientists and they often wanted to quit the study (Epstein, 1997; Oudshoorn, 2003).

\section{Overcoming Recruitment and Retention Obstacles through Tuning Work}

Pickering (1995) saw the relationship between human and non-human elements as a dance of agency, where the material objects can resist the work of scientists. To address this resistance, scientists tune their instruments after which they assess how the instruments respond to the modifications. Griswold et al. (2013, p. 348) noted that dance of agency as a metaphor 'foregrounds interaction and broadens participation as it connects movement through space and mutual adjustment over time'.

There are several studies that have utilized Pickering's tuning concept to understand the interactions between human and non-human actors (e.g., Griswold et al., 2013; Martini et al., 2013; Brenninkmeijer, 2013). A study by Barrett et al. (2012), which examined the installation of a robot in a hospital pharmacy, is useful for understanding tuning work among the research actors. Barrett $e t$ al. (2012, p. 1451) described the hospital pharmacy as a context that is 'rarely constituted by single human actors but rather include multiple groups of actors, thus entailing a diversity of (often conflicting) interests, values, norms, competencies, and practices'.

While the study shed light on both human and material agency, it highlighted interaction, participation, and adjustment over time among three groups of professionals: pharmacists, technicians, and assistants (Barrett et al., 2012). They organized planning meetings, where pharmacists and technicians tuned the robotic innovation to their varied interests and intentions. Similarly to the meetings where the research actors figured out how to address recruitment obstacles, 
the meetings on the implementation of the robot functioned as spaces for resistance and accommodation (Barrett et al., 2012).

The following analysis of meeting interactions focuses on the question of who tuned work practices, and how, when faced with a recruitment obstacle. While the research actors adjusted their work practices, personal routines, and the study protocol to ease the problem of cultural taboos, the burden of long-term commitment provided fewer opportunities for tuning work and highlighted the professional boundary between the scientists and the study coordinators. Therefore, it remained as a more permanent recruitment obstacle than the problem of cultural taboos.

\section{Addressing the Problem of Cultural Taboos}

Once it was established through focus group interviews and interactions with patients in the clinic that participation in the human microbiome study was a tough sell for potential participants, the research actors began to think about ways in which the problem of cultural taboos could be eased. The physicians, the study coordinators, and the scientists were all willing to adjust their work practices in some way to bypass the embarrassment associated with fecal samples. These adjustments can be seen as 'strategies of accommodation' developed for achieving the intended outcomes (Martini et al., 2013, p. 200).

Because the dispensing robot was implemented in the hospital pharmacy without input from the assistants, they perceived the changes to the organization of pharmacy work as undermining the assistants' work activities and symbolizing their lower professional status (Barrett et al., 2012). In contrast, the physicians of the present study had a high professional status to start with and the recruitment obstacles discussed above only highlighted their important role in the recruitment setting, the university's obstetrics and gynecology clinic. At a meeting focused on the difficulties associated with recruiting subjects at the clinic, one senior physician suggested that he would advise the fellows 
and residents working with him to talk to their patients about the human microbiome study. He was aware that they had power to influence how their patients behaved.

So would it be helpful if we had a script that we could give to the fellows and the residents [at the clinic] saying, 'Has somebody talked to you about some of the studies we are doing?' Because patients will often listen to us. Particularly if they are our return patients. I have a host of patients who are my returns who are just very happy to participate. (ID-7; 052312)

In response, one of the study coordinators addressed the physicians in the room, saying, 'If you guys could all do that, I think that would make a huge difference' (ID-35).

As the physicians were invited to the meetings already at the planning stage, they developed a thorough understanding of the human microbiome project. Therefore, they were able to imagine how they could adjust their practices and thus contribute to the recruitment effort. Differently, in the study by Barret et al. (2012), assistants were not included in the planning of how the robot should be implemented in the pharmacy. Once the robot was installed, it didn't fit in with the assistants' work practices and, as a result, they developed a negative attitude towards the technology. The physicians, however, were supportive of the human microbiome project. Incorporating an introduction to the human microbiome study into the regular physician-patient interactions was not a big adjustment from their perspective, but it significantly eased the problem of transgressing cultural taboos.

Furthermore, the physicians had close, trusted relations with the expectant mothers and they were used to discussing sensitive bodily matters with them throughout pregnancy. In fact, Lappé (2014) has emphasized the value of intimate relationships between research and care in her study on the conduct of medical research on autism. This close relationship was extremely valuable for the recruitment effort, because anything related to defecation is not considered a socially acceptable 
conversation topic and even when it is discussed, people tend to use euphemisms and circumlocutions (Inglis, 2000; Weinberg and Williams, 2005). Thompson (2013) found that those suffering from inflammatory bowel disease had an easier time talking about their condition in support group meetings if the discussions remained clinical. The physicians talking about the sample collection effort in a clinical context could ease feelings of shame and embarrassment. While discussing bodily matters is often perceived as taboo, sharing such details as they relate to physical ailments is normal in clinical settings. 'Physicians may not pass judgment and must practice discretion,' as Thompson (2013, p. 31) pointed out.

The study coordinators did not have the same kind of professional authority and established close relations with the women as the physicians. One study coordinator realized, however, that there was a way in which she could adjust her life so as to help the recruitment effort. When she became pregnant, she enrolled in the study herself. In a biannual newsletter reporting on the medical research center's scientific efforts, the study coordinator was interviewed as part of an article on how the research project's subject recruitment was progressing. The study coordinator described how she used her own experiences with self-sampling and storing different bodily substances as an entry point when she approached patients at the clinic.

Rather than tuning her work practices in relation to recruitment obstacles like the physicians did, the study coordinator adjusted her personal life to benefit the recruitment effort. She experienced firsthand what it was like to participate in the study and challenge established excretory practices and notions of fecal habitus (Inglis, 2000). The study coordinator said that when recruiting new subjects, being able to tell the women that she too had collected weekly samples of feces and other bodily substances and showing them that she was still all right, made a huge difference. It is worth noting that this example of tuning work was done without any professional dependency to the other research 
actors. At least this one study coordinator seemed to have agency, which the assistants felt they were lacking after the dispensing robot was installed in the hospital pharmacy (Barrett et al., 2012).

While the physicians and the study coordinators were proactive about tuning their work practices or even their personal life to ease the problem of cultural taboos, the scientists remained in the background. This might relate to the power difference between the scientists and the study coordinators, who, as two entities, were differently obligated by the research project (Callon, 1986). The scientists may have seen the study coordinators as the promoters of their sample collection protocol.

The situation changed, when the study coordinators made the argument that in order to ease the problem of breaking the cultural taboos associated with handling the fecal samples, the study protocol should be adjusted by including rectal swabs as one sample collection method. A member of the research staff told the scientists, 'I suspect that there will be patients who would consent to doing a rectal swab but who won't consent to catching stool' (ID-23; 091411).

In the mangle of practice, resistance can be offered by any entity, no matter what's their formal role in knowledge production (Pickering, 1993). Although the study coordinators found it challenging to recruit women to a study that transgressed cultural taboos, they couldn't modify the study protocol without the scientists' support. A similar dependency between professional groups was present in the study by Barrett et al. (2012, p. 1458), where assistants perceived a loss of control of in their work when technicians were seen as the 'caretaker of technology' and the only ones equipped with digital passwords to resolve problems that occurred with the robot.

The suggestion to use rectal swabs can be seen as a strategy for moderating the fear of pollution (Douglas, 1966). By using rectal swabs as opposed to 'catching stool,' the subjects did not have to look at fecal material. This suggestion led to an exchange between two scientists, who were unsure if rectal swabs would provide them with the data they needed. 
ID-5: I think what we need to do in our lab is a test of how much DNA can be obtained from a rectal swab for the purposes of all the downstream stuff we want to do with it, because I don't think we really have a good idea.

ID-24: A fecal sample is probably preferable, just because of the amount. (091411)

The conversation on whether rectal swabs had enough DNA continued for months. At a later meeting one scientist noted:

There may be women who are unwilling to really put the swab in. They may sort of touch some place on the butt and we will end up having a skin sample. The real problem is that we could easily end up with a skin sample rather than a fecal sample. So that's why ideally it would be - if you can do a poop, great, but if you can’t, it's a swab. (ID-24; 051612)

The scientists thus had a couple of concerns regarding rectal swabs. First, they were unsure if rectal swabs would contain enough DNA for further analysis. Second, they feared that research subjects would collect skin rather than fecal samples.

After several meeting discussions on the benefits of fecal samples versus rectal swabs, with the study coordinators sharing stories of subjects refusing to participate or leaving the study midway, the scientists began to agree that the study protocol would have to be adjusted. While the scientists feared that rectal swabs would not always provide them with the data they needed (e.g., skin versus fecal sample), they saw that it was necessary to give the study participants some flexibility over how they conducted the self-sampling procedure. In this way, the human microbiome project meetings functioned as spaces for resistance and accommodation among different professionals (Barrett et al., 
2012). This is also an example of how study protocols are not 'immutable mobiles' (Latour, 1990). The research actors, their work practices, and the study protocol began to supplement each other over the course of the group meetings (Lynch, 2002).

Both the physicians and the study coordinators tuned their work practices in order to address the problem of cultural taboos. By introducing the human microbiome study to women in the clinical context or sharing their own experiences with it, the physicians and the study coordinators demonstrated that it was possible to approach the sample collection effort without shame. Yet the most effective strategy for moderating the fear of pollution was the modification to the study protocol with regards to how fecal samples could be collected. This piece of tuning work required negotiation between scientists and study coordinators, as the scientists-similarly to the technicians who had the power to resolve technological problems with the robot—had privileged access to modify the study protocol (Barrett et al., 2012). Over time, the study coordinators convinced the scientists that it was worthwhile to give participants some choice over how to collect the fecal sample.

\section{Addressing the Burden of Long-Term Participation}

As the scientists sought to understand how women's bacterial communities evolved during pregnancy, and stabilized after delivery, a longitudinal study design was a necessity. While the longterm commitment made the enrollment of new subjects challenging, re-engaging enrolled participants after they had given birth was particularly difficult. After becoming mothers, the study participants' life and daily routines changed and their interests no longer aligned with those of the research team (Epstein, 1997; Oudshoorn, 2003).

This did not come as a surprise to the study coordinators and the research staff who, from the start, saw the longitudinal time perspective as a challenge for recruiting and retaining subjects. When planning the study protocol, the research actors discussed about how many months after delivery the 
study participants might realistically be willing to continue sampling and storing their bodily substances. In these meetings on the length of study participation time, there was resistance from the side of the study coordinators and the research staff and, in turn, not much accommodation from the side of the scientists (Pickering, 1995; Barrett et al., 2012).

In the beginning of the project, it was decided that the subjects would be asked for only one set of samples collected six weeks after they had given birth. Soon after, the study protocol was made more extensive, because the scientists argued that one data point was not enough to examine how bacterial communities stabilized after delivery. The study protocol was changed accordingly; the women were now asked to continue weekly self-sampling for three months after delivery.

A few months into the recruitment effort, the research staff discovered that the scientists had further modified the protocol without consulting them. The protocol now requested that the weekly self-sampling of different body sites and bodily substances be continued for twelve months after delivery. The study coordinators were not happy about this modification and resisted, rather than accommodated, the scientists' modification to the protocol (Pickering, 1993). While in Pickering's account, human plans and activities are transformed during interactions with technologies, here the study coordinators' perspective on the length of study participation time was shaped by their interactions with the research subjects (Pickering, 1995). As one of them noted:

The people I have approached, they have been like 'okay, mm-hmm, yeah,' and then you say, 'Oh and we will also collect samples for up to a year after delivery,' and they look at you and they are like, 'A year? Really? I don't know.' It's just the thing I kind of dread telling them. (ID-38; 032812)

A member of the research staff addressed the scientists as follows: 
ID-23: I thought the study was ending when the women delivered and we got their six-week self-sampling, then it went to three months and now it seems to have gone for a year out. Our study coordinators think that that's a deterrent, that the women might be more accepting if it was at three months, say, what we are expecting of them and for how long.

ID-24: Maybe we shouldn't ask them upfront then. Later on, they may feel like they are now kind of a member of the family. Because the postnatal stuff beyond three months is not critical to our study of pregnancy. We don't need to pose it upfront, and if it has any detrimental impact, then we definitely should not, but by the time we get close to delivery it's not bad; just sort of approach the issue again, say[ing] 'This is totally optional. You can say no, but we are interested.' (032812)

Tuning work, while here mainly seen as adjustments to different professionals' work practices, also relates to material objects—-for instance a dispensing robot—around which different work tasks take place (Barrett et al., 2012). For the study coordinators, the protocol was a documentor a material object - that determined how they explained the study and its requirements to potential participants. It helped them to describe the sample collection procedure and answer participants' questions accurately. The protocol was like an immutable mobile for the study coordinators (Latour, 1990). The content of the document was stable and stayed the same for all the women they approached. This was a critical aspect of the study coordinators' work and they emphasized that it wasn't acceptable to modify the protocol without at least notifying them in advance.

The scientists had a more flexible understanding of the study protocol, especially with regards to how and when the long-term commitment was explained to the women participants. In the 
discussion extract above, for example, the scientist suggested that the study coordinators should introduce the topic of ongoing study participation after delivery, or 'later on,' not during the first enrollment conversation. The women might oppose the longitudinal study design less when the selfsampling procedure had become part of their weekly routines. The scientist said that by the time the women gave birth, '[T]hey may feel like they are now kind of a member of the family.' Like the laboratory protocols Lynch (2002) analyzed, the scientists saw the implementation of study protocols as a lively combination of local practices and written inscription.

To summarize, perhaps the biggest challenge for tuning work is when one occupational group tries to dictate how another occupational group should conduct their work in relation to shared goals. By excluding the assistants from the planning stage, the technicians and the pharmacists did not consider how the dispensing robot would influence the assistants' work. The tuning process associated with implementing the dispensing robot symbolized 'pain for the assistants and gain for the technicians and pharmacists' (Barrett et al., 2012, p. 1459).

Along the same lines, the scientists were not willing to shorten the length of study participation time. To address the burden of long-term commitment and its effect on retention, they proposed instead that the study coordinators tuned their work practices in an effort to accommodate the scientists' research goals. In practice, the study coordinators were asked to modify when they introduced long-term commitment to study participants. The study coordinators, on the other hand, did not appreciate the suggestion as it meant concealing some aspects of the study protocol and jeopardizing research ethics.

\section{Conclusion}

This longitudinal qualitative study addressed the research question of how research actors moderate feelings of embarrassment and intrusion resulting from cultural taboos and the longitudinal 
nature of human microbiome studies. The research actors experienced the presence of cultural taboos against collecting the required research materials, and the need to collect long-term data as recruitment obstacles. They sought to ease these obstacles by tuning their practices towards shared recruitment goals. Pickering's (2005) conceptualization of tuning, typically seen as the reciprocal adjustment of human and material agency, helped in analyzing this collaborative process. The research actors sought to tune their work practices in order to moderate research subjects' feelings of embarrassment and intrusion caused by the study protocol.

While some social scientists have studied how research actors use their professional roles in the generation of long-term participation in medical research (e.g., Lappé, 2014), there are hardly any studies on how research actors gather data on bodily substances that participants perceive as culturally taboo. As human microbiome researchers want to collect samples of bodily substances such as feces and urine, they need to cross the boundary between study participants' public and private bodies (Thompson, 2013). Douglas's (1966) conceptualizations of taboos, dirt, and matter out of place helped to illuminate the challenges involved in crossing this boundary. The study protocol threatened the individuals' fecal habitus as well as the everyday social order by requesting participants to catch their stool in containers and then store it in their freezers (Inglis, 2000; Weinberg and Williams, 2005).

The generation of long-term participation was a recruitment and retention obstacle in this context, because the study participants would have to continue the weekly self-sampling procedure after a significant life change: becoming a mother. Giving birth can be seen as a transitional state, in which it is unknown what the individual will experience and how it will change her (Douglas, 1966; Stern et al., 1998). The scientists hoped that the longitudinal time perspective would ease the burden of long-term participation, because over time the sample collection procedure would become part of the women's weekly routines. As such, longitudinal participation would come naturally. This sense of a routine would help the research team to convince the women also to participate after delivery. 
Yet it wasn't easy for the study coordinators to re-engage the new mothers in the sample collection effort. Requesting the continuation of weekly self-samplings from them felt like crossing another kind of boundary between public and private aspects of study participants' lives.

Extending Pickering's (1995) tuning approach, I demonstrated how some aspects of these two recruitment and retention challenges could be eased through tuning work. While Pickering's dance of agency has been typically used to analyze the relationship between non-human and human actors (e.g., Griswold et al., 2013; Martini et al., 2013; Brenninkmeijer, 2013), I used the conceptualization of tuning to understand adjustments to, in particular, how the research actors conducted their work.

Firstly, the research actors adjusted their work practices, personal routines, and the study protocol in order to bypass the presence of cultural taboos. The physicians and the study coordinators adjusted how they talked to the women about the sample collection effort. By doing so, they sought to demonstrate that typical excretory practices, such as correct ways of defecating and talking about excrement, can in certain contexts be transformed (Inglis, 2000). Catching and storing one's stool for scientific purposes was acceptable and not a threat to the fecal habitus (Inglis, 2000; Weinberg and Williams, 2005). The most important strategy for addressing the problem of cultural taboos was, however, the inclusion of rectal swabs as one sample collection method in the study protocol, which can be seen as an adjustment to a material object (Pickering, 1995). After this modification, the subjects no longer had to see their fecal material and have containers filled with feces in their freezers.

Secondly, tuning work seemed less influential for trying to address the burden of long-term participation. The ability of the physicians and the study coordinators to engage in tuning work was limited, because it was not obvious how they could bypass the recruitment and retention obstacle by adjusting their work practices. Modifications to the length of study participation time would have to be made in the domain of the scientists' work. The scientists did not want to make the sample collection effort less extensive, so they suggested that the study coordinators introduced the long- 
term commitment later on when the women felt comfortable about self-sampling their bodily substances on a regular basis.

This was a problem for the study coordinators who saw the protocol as a document that identified what was required of study participants. In Lynch's (2002, p. 204) words, the document was meant to 'describe the observational procedure without adding any interpretation.' The study coordinators could adjust how they talked about the self-sampling of fecal material and even enroll in the study to have a shared experience with the participating women. They could not, however, conceal what the study protocol asked of subjects when they first interacted with the women at the clinic. This would be an ethical violation.

Pickering (1995) argued that in the dance of agency when machines resist scientific experiments, scientists revise their goals and practices. In other words, the technological and the social aspects of knowledge creation find a balance through a process of trial and error. Based on the instances of tuning work that this study uncovered, it seems that scientists react to resistance from study participants differently than they do to resistance from technological instruments. In The Mangle of Practice (Pickering, 1995), the scientist assembled a set-up, stood back to see what happened, reconfigured the apparatus, again observed what happened, reassembled the set-up, and so on. In relation to the study participants' refusal to participate, the human microbiome researchers seemed reluctant to adjust their machine: the study protocol. In relation to the year-long commitment after delivery, the scientists thought it was best to wait and see, hoping that the women would change their minds or overcome feelings of intrusion. Overall, it seemed as though the physicians and the study coordinators were more likely to react to resistance from study participants.

This study shed light on how research actors prepare for the implementation of demanding study protocols and how they manage recruitment issues that emerge. This collaborative process can be best described as tuning work. That is, research actors modify, for instance, their work practices 
and details of the study protocol in order to make the data collection effort more agreeable to research subjects. To the public and potential research subjects, tuning work is mostly invisible as it involves adjustments that are discussed and decided on behind closed doors. When a study coordinator arrives at a clinic with a consent form and a script for how to approach subjects, it is impossible to assess how tuning work influenced the recruitment process.

In most cases, tuning work probably involves harmless adjustments to how professionals conduct their work. However, when physicians, their relationships to patients, and knowledge of how the clinic works are used in figuring out how to recruit research subjects, ethical concerns surface. Are there boundaries that should not be crossed when research actors engage in tuning work? Should study participants be aware of how tuning work is used in recruitment and retention? For those of us who study recruitment and retention, getting access to see what happens behind closed doors as research actors develop 'strategies of accommodation' should be a priority (Martini et al., 2013, p. 200).

\section{Acknowledgements}

I am grateful to the reviewers and the editors for their detailed feedback, as well as to Dan McFarland, Woody Powell, John Willinsky, and Karen Cook for their comments on the very first draft. I would also like to thank the members of my wonderful online writing group: Charlie Gomez, Ben Keep, and Sebastian Munoz-Najar Galvez.

\section{Disclosure Statement}

No potential conflict of interest was reported by the author. 


\section{Funding}

The study is part of a larger project titled Transdisciplinary Science and Premature Birth. As part of this project, the author received a PhD scholarship from the March of Dimes Foundation 2011-2015.

\section{Biographical Note}

Elina I. Mäkinen is an organizational researcher specializing in collaboration and innovation in the life sciences. Dr. Mäkinen received her Ph.D. in Stanford University's organizational studies doctoral program. She was recently appointed as an Associate Professor in the New Social Research Program at the University of Tampere, Finland.

\section{References}

Barrett, M., Oborn, E., Orlikowski, W. J. and Yates, J. (2012) Reconfiguring boundary relations: Robotic innovations in pharmacy work, Organization Science, 23(5), pp. 1448-1466.

Brenninkmeijer, J. (2013) Neurofeedback as a dance of agency, BioSocieties, 8(2), pp. 144-163.

Cahill, S. (2006) Building bodily boundaries. Embodied enactment and experience, in: D. Waskul and P. Vannini (eds.) Body/Embodiment: Symbolic Interaction and the Sociology of the Body, 1st ed., pp. 69-82 (Burlington: Ashgate).

Callon, M. (1986) Some element of a sociology of translation: Domestication of the scallops and the fishermen of St Brieuc Bay, in: J. Law (ed.) Power, Action, and Belief: A New Sociology of Knowledge? 1st ed., pp. 196-233 (London: Routledge \& Kegan Paul). 
Curtis, V. A. and Biran, A. (2001) Dirt, disgust, and disease: Is hygiene in our genes?, Perspectives in Biology and Medicine, 44(1), pp. 17-31.

Douglas, M. (1966) Purity and Danger: An Analysis of Concepts of Pollution and Taboo, 1st ed. (London: Routledge \& Kegan Paul).

Elias, N. (2000) The Civilizing Process: Sociogenetic and Psychogenetic Investigations, revised ed. (Oxford: Wiley-Blackwell Publishers).

Epstein, C. F. (1992) Tinker-bells and pinups: The construction and reconstruction of gender boundaries at work, in: M. Lamont and M. Fournier (eds.) Cultivating Differences: Symbolic Boundaries and the Making of Inequality, 1st ed., pp. 232-256 (Chicago: University of Chicago Press).

Epstein, S. (1997) Activism, drug regulation, and the politics of therapeutic evaluation in the AIDS era: A case study of ddC and the 'surrogate markers' debate, Social Studies of Science, 27(5), pp. $691-726$.

Epstein, S. (2008) Clinical research, racial knowledge, and the politics of inclusion and difference, Social Studies of Science, 38(5), pp. 801-832.

Gabillet, A. (2017) I Sent Away a Sample of My Poop, and Here's What I Learned About My Health, Popsugar.com, October 3. Available at https://www.popsugar.com/fitness/How-Get-YourGut-Bacteria-Analyzed-44053860 (accessed 15 April 2018). 
Gee, J. P. (2005) An Introduction to Discourse Analysis: Theory and Method, 2nd ed. (London: Routledge).

Goffman, E. (1967) Interaction Ritual: Essays on Face-to-Face Behavior, 1st ed. (New York: Anchor Books).

Goodwin, C. (2000) Action and embodiment within situated human interaction, Journal of Pragmatics, 32(10), pp. 1489-1522.

Griswold, W., Mangione, G. and McDonnell, T. E. (2013) Objects, words, and bodies in space: Bringing materiality into cultural analysis, Qualitative Sociology, 36(4), pp. 343-364.

Inglis, D. (2000) A Sociological History of Excretory Experience: Defecatory Manners and Toiletry Technologies, 1st ed. (Lewiston: Edwin Mellen Press).

Lappé, M. D. (2014) Taking care: Anticipation, extraction and the politics of temporality in autism science, BioSocieties, 9(3), pp. 304-328.

Latour, B. (1987) Science in Action: How to Follow Scientists and Engineers through Society, 1st ed. (Cambridge: Harvard University Press).

Latour, B. (1990) Drawing things together, in: M. Lynch and S. Woolgar (eds.) Representation in Scientific Practice, 1st ed., pp. 19-68 (Cambridge: MIT Press). 
Lynch, M. (2002) Protocols, practices, and the reproduction of technique in molecular biology, British Journal of Sociology, 53(2), 203-220.

Martini, A., Massa, S. and Testa, S. (2013) The firm, the platform and the customer: A 'double mangle' interpretation of social media for innovation, Information and Organization, 23(3), 198213.

Mueller, M. R. (1997) Science versus care: Physicians, nurses, and the dilemma of clinical research, in: M. A. Elston (ed.) The Sociology of Medical Science and Technology, 1st ed., pp. 57-78 (Oxford: Blackwell Publishers).

Oudshoorn, N. (2003) The Male Pill: A Biography of a Technology in the Making, 1st ed. (Durham: Duke University Press).

Palmer, C. K., Thomas, M. C., von Wagner, C. and Raine, R. (2014) Reasons for non-uptake and subsequent participation in the NHS Bowel Cancer Screening Programme: A qualitative study, British Journal of Cancer, 110(7), pp. 1705-1711.

Palmer, C. K., Thomas, M. C., McGregor, L. M., von Wagner, C. and Raine, R. (2015) Understanding low colorectal cancer screening uptake in South Asian faith communities in England-A qualitative study, BMC Public Health, 15:998. 
Pickering, A. (1993) The mangle of practice: Agency and emergence in the sociology of science, American Journal of Sociology, 99(3), pp. 559-589.

Pickering, A. (1995) The Mangle of Practice: Time, Agency, and Science, 1st ed.(Chicago: University of Chicago Press).

Pickering, A. (2005) Decentering sociology: Synthetic dyes and social theory, Perspectives on Science 13(3), pp. 352-405.

Pickersgill, M. (2012) The co-production of science, ethics, and emotion, Science, Technology, \& Human Values, 37(6), pp. 579-603.

Rozin, P. and Fallon, A. (1987) A perspective on disgust, Psychological Review, 94(1), pp. 23-41.

Rozin, P., Haidt, J. and McCauley, C. R. (1993) Disgust, in: M. Lewis and J. M. Haviland (eds.) Handbook of Emotions, 1st ed., pp. 575-94 (New York, NY: The Guilford Press).

Sidnell, J. (2007) Comparative studies in conversational analysis, Annual Review of Anthropology, 36, pp. 229-244.

Stern, D. N., Bruschweiler-Stern, N. and Freeland, A. (1998) The Birth of a Mother: How the Motherhood Experience Changes You Forever, 1st ed. (New York: Basic Books).

Synnott, A. (1993) The Body Social: Symbolism, Self, and Society, 1st ed. (London: Routledge). 
Thompson, A. I. (2013) 'Sometimes, I think I might say too much’: Dark secrets and the performance of inflammatory bowel disease, Symbolic Interaction, 36(1), pp. 21-39.

Weinberg, M. S. and Williams, C. J. (2005) Fecal matters: Habitus, embodiments, and deviance, Social Problems, 52(3), pp. 315-336. 